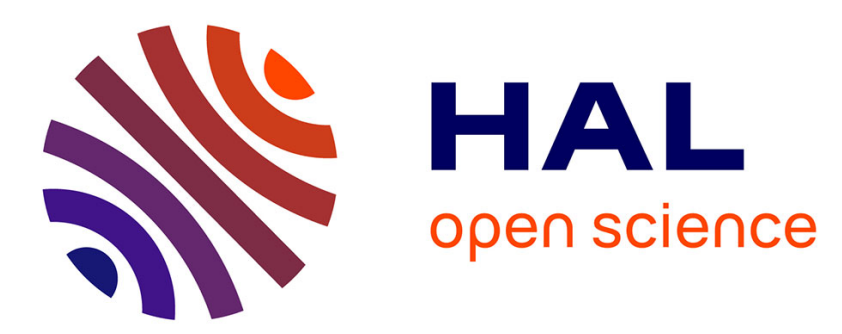

\title{
Electronic Spectrum of a 2D Quasi-Crystal Related to the Octagonal Quasi-Periodic Tiling
}

\author{
Clément Sire
}

\section{To cite this version:}

Clément Sire. Electronic Spectrum of a 2D Quasi-Crystal Related to the Octagonal Quasi-Periodic Tiling. EPL - Europhysics Letters, 1989, 10, pp.483-488. 10.1209/0295-5075/10/5/016 . hal00472630

\section{HAL Id: hal-00472630 \\ https://hal.science/hal-00472630}

Submitted on 12 Apr 2010

HAL is a multi-disciplinary open access archive for the deposit and dissemination of scientific research documents, whether they are published or not. The documents may come from teaching and research institutions in France or abroad, or from public or private research centers.
L'archive ouverte pluridisciplinaire HAL, est destinée au dépôt et à la diffusion de documents scientifiques de niveau recherche, publiés ou non, émanant des établissements d'enseignement et de recherche français ou étrangers, des laboratoires publics ou privés. 
Electronic Spectrum of a 2D Quasi-Crystal Related to the Octagonal Quasi-Periodic Tiling

This article has been downloaded from IOPscience. Please scroll down to see the full text article.

1989 Europhys. Lett. 10483

(http://iopscience.iop.org/0295-5075/10/5/016)

The Table of Contents and more related content is available

Download details:

IP Address: 130.120.231.223

The article was downloaded on 12/04/2010 at 16:35

Please note that terms and conditions apply. 


\title{
Electronic Spectrum of a 2D Quasi-Crystal Related to the Octagonal Quasi-Periodic Tiling.
}

\author{
C. SIRE \\ Laboratoire de Physique des Solides-C.N.R.S. \\ 1, place Aristide Briand, 92195 Meudon, France
}

(received 26 June 1989; accepted in final form 16 August 1989)
PACS. 71.20 - Electronic density of states determinations (inc. energy states of liquid semiconductors).

PACS. 71.25C - Techniques of band-structure calculation (general theory, applications of group theory, analytic continuation, etc.).

\begin{abstract}
Recently, a tiling derived from the well-known 2D quasi-periodic octagonal tiling has been introduced. In this letter, we show that in the framework of a tight-binding model, the electronic spectrum of this nontrivial tiling can be derived. The integrated density of state is singular and can be a devil staircase, there can be a finite or infinite number of gaps, whereas the measure of the spectrum can be zero or not, all these properties depending on the hopping parameters. This transition is explained with a very simple model.
\end{abstract}

\section{Introduction.}

Since the discovery of quasi-crystals by Schechtman et al. [1], many authors are interested in the study of the electronic spectrum in solids with a quasi-periodic structure, in one, two or three dimensions. In all dimensions, there are a lot of numerical works, which shows that the electronic spectrum of a quasi-crystal has a singular part [2]. The 1D case has been intensively studied [3-5], whereas in $2 \mathrm{D}$ or $3 \mathrm{D}$, there are very few exact results, except for superlattices built from the Fibonacci sequence studied by Ueda and Tsunetsugu [6]. In the following, we introduce a tiling closely related to the $2 \mathrm{D}$-octagonal quasi-periodic tiling, and study its spectrum.

\section{The labyrinth.}

Recently, a new tiling, we call it the labyrinth, has been introduced [7]. It is built univoquely from the quasi-periodic octagonal tiling. More precisely, it can be considered as a subset of the octagonal tiling, and conversely. It is built with a kite, a trapezoid and a square whose dimensions are functions of a certain parameter $\alpha$ (see [7] for more details). For $\alpha=1+\sqrt{2}$, we obtain the labyrinth which can be found directly, starting from the octagonal quasi-periodic tiling. We show the labyrinth in fig. 1, for different values of $\alpha$. The origin of its name is manifest. We give the two main results which will be useful in the following. The coordinates of the vertices of the labyrinth $(\alpha=1+\sqrt{2})$ can be exactly derived and can be 


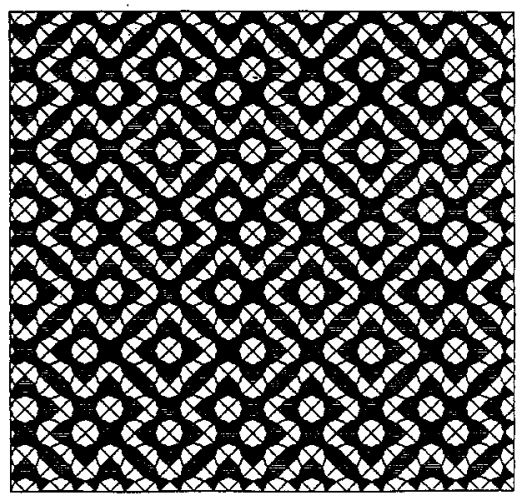

a)

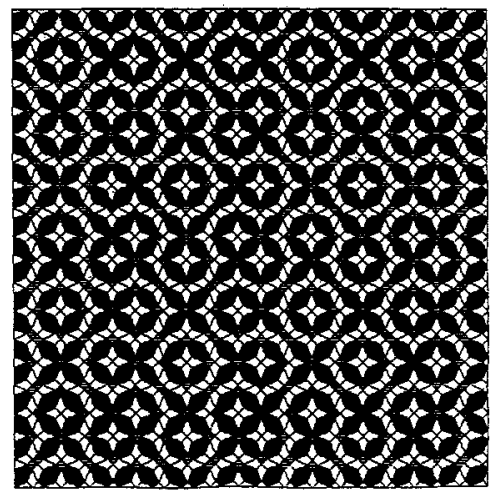

b)

Fig. 1. - The labyrinth for different values of $\alpha$. a) $\alpha=1+\sqrt{2}, b) \alpha<1$ (here, $\alpha=\sqrt{2}-1$ ).

written as

$$
\left\{\begin{array}{l}
X_{l, m}=l+(F((l+m) / \sqrt{2})+F((l-m) / \sqrt{2})) / \sqrt{2}, \\
Y_{l, m}=m+(F((l+m) / \sqrt{2})-F((l-m) / \sqrt{2})) / \sqrt{2},
\end{array}\right.
$$

where $F(x)$ is the closest integer to $x$ and $l, m \in Z$. It is clear from (1) that the labyrinth has an average lattice (which is a square lattice), by taking $F(x)=x$.

Moreover, it can be shown that the labyrinth can be obtained in a different way. Let us consider $S_{l}$, a sequence of letters $L$ and $S$ (the «Octonacci» sequence), obtained by means of the following recursion formula:

$$
S_{0}=S, \quad S_{1}=L, \quad S_{l+2}=S_{l+1} * S_{l} * S_{l+1} \quad \text { and } \quad S_{\infty}=\lim _{l \rightarrow \infty} S_{l}
$$

where $*$ is a symbol for chain concatenation. One can associate a linear chain to $S_{l}$ (we call it $R_{l}$ ) by associating a length 1 to a $S$ and a length $\alpha$ to a $L$, and symmetrizing it with respect to the origin. Now, we build the Euclidean product $R_{\infty} \times R_{\infty}$, and remove all vertices which cannot be linked to the origin by means of diagonal bonds (that is nonvertical nor horizontal bonds). Finally, if we link nearest neighbours by diagonal bonds, we recover the labyrinth

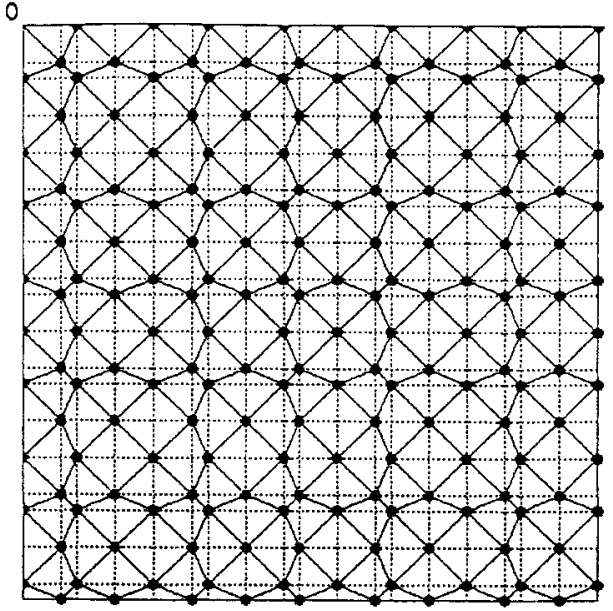

Fig. 2. - The labyrinth as it is built in sect. 3-4. The dotted lines represent the underlying Euclidean product. 
(fig. 2). This last method will be very useful in order to derive the electronic properties of the labyrinth.

\section{The product of two linear chains.}

Let us consider two linear chains of atoms, $S_{1}$ and $S_{2}$. One can study the electronic spectrum of each chain in the framework of a tight-binding model. We suppose that on $S_{i}$, $i=1,2$, the hopping parameter between nearest neighbours can take only two values, $s_{i}$ and $l_{i}$. Of course, this condition is not necessary and the following calculation is not affected by a biggest set of values for the hopping terms. Moreover, for $S_{i}$, we take the diagonal terms of the Hamiltonian equal to $v_{i}, i=1,2$. Now, suppose we know the electronic spectrum and the wave functions for each linear chain:

$$
\left\{\begin{array}{l}
t_{i, j} \Phi_{i, j-1}+v_{i} \Phi_{i, j}+t_{i, j+1} \Phi_{i, j+1}=\left(E_{i}+v_{i}\right) \Phi_{i, j}, \\
t_{i, j} \in\left\{s_{i}, l_{i}\right\}, i=1,2, j \in Z
\end{array}\right.
$$

where $E_{i}+v_{i}$ is in the spectrum of $S_{i}$, and $\Phi_{i, j}$ is the eigenvector value at the site $j$ of $S_{i}$. By doing the product of two such equalities (one for each chain), we obtain

$$
\sum_{n, n} v_{l, m} h_{l, m}=E h_{i, j}
$$

with

$$
\begin{gathered}
E=\left(\left(E_{1}+v_{1}\right)\left(E_{2}+v_{2}\right)-v_{1} v_{2}\right) / V, \quad h_{i, j}=\Phi_{1, i} \Phi_{2, j} \\
V v_{l, m} \in\left\{s_{1} s_{2}, l_{1} l_{2}, s_{1} l_{2}, s_{2} l_{1}, v_{1} s_{2}, v_{2} s_{1}, v_{1} l_{2}, v_{2} l_{1}\right\}
\end{gathered}
$$

and the sum holds on the eight nearest neighbours of the site $(i, j)$. We introduced an arbitrary constant $V$ whose dimension is an energy, for $E$ to have the same dimension. We see that the eight hopping parameters are not independent, since they are functions of only six independent ones. So, we can build the spectrum of a lattice with rather complicated interactions, provided we can find linear chains whose spectrum is known, starting from which the lattice can be built. The same calculation can be done in $3 \mathrm{D}$ or with second nearest neighbours.

Let us study two particular cases. The first one is well known, and corresponds to the case where we take $v_{1}=v_{2}=V$ and $V=+\infty$. Then, we obtain the usual Euclidean product of the linear chains. Of course, in that case, the formula (3) for the energy gives $E=E_{1}+E_{2}$, which is a classic result. A more interesting result is obtained when taking $v_{1}=v_{2}=0$. In that case, one can see that vertices linked by a vertical or horizontal bond in the Euclidean product $S_{1} \times S_{2}$ do not interact any more, since the hopping parameter for such bonds is in the set $\left\{v_{1} s_{2}, v_{1} l_{2}, v_{2} s_{1}, v_{2} l_{1}\right\}$. More precisely, if we link together every couple of interacting sites on $S_{1} \times S_{2}$, we obtain two superimposed and connected set of vertices, each of them being the dual of the other one (fig. 2). If we take one vertex of $S_{1} \times S_{2}$ as the origin, we can see that the first lattice contains vertices whose coordinates have the same parity, while the other one consists in vertices with coordinates with opposite parity. Even if the resulting tiling is not necessarily identical to its dual, they have the same spectrum, since (2) and (3) split in two disconnected sets of equations. Nevertheless, there is no reason for a simple correspondence between both wave functions. Moreover, the spectrum of each lattice is the set $\left\{E_{1} E_{2}, E_{1} \in \operatorname{Sp}\left(S_{1}\right), E_{2} \in \operatorname{Sp}\left(S_{2}\right)\right\}$, where $\operatorname{Sp}\left(S_{i}\right)$ is the spectrum of $S_{i}$.

In the next section, we show that this calculation can be applied to the labyrinth. 


\section{The electronic spectrum of the labyrinth.}

In (1), let us define $p$ and $q$ by $p=l+m$ and $q=l-m$. Then, the coordinates can be expressed in an equivalent way by

$$
\text { - }\left\{\begin{array}{l}
X_{p, q}=U_{p}+U_{q}, \quad Y_{p, q}=U_{p}-U_{q}, \quad p=q \bmod 2 \\
U_{p}=p / 2+F(p / \sqrt{2}) / \sqrt{2}
\end{array}\right.
$$

Moreover, it can be shown that the sequence of long and short lengths along the chain $U=\left\{U_{p}, p \in Z\right\}$ is identical to $R_{\infty}$. Thus, the labyrinth consists in the vertices of the Euclidean product $U \times U$ whose coordinates have the same parity (fig. 2). According to sect. 3 , if we know the spectrum of the linear chain $U$, we also know the electronic spectrum of the labyrinth and its dual. So, let us study the spectrum of $U$. Since $U$ has the same structure as $R_{\infty}$, which follows the Octonacci sequence, we first study the spectrum of a crystal of elementary cell $R_{l}$, before taking $l$ infinite. Then, our problem becomes very similar to a tight-binding model on the Fibonacci chain. We expect to find a trace mapping whose iterations will lead to the spectrum. Indeed, very similar calculations as those done in [4] give the following result. Let the hopping parameter be 1 , for a long bond, $r$, for a short one. We define $M_{1}(x)$ and $M_{2}(x)$ by

$$
M_{1}(x)=\left|\begin{array}{cc}
x & -1 \\
1 & 0
\end{array}\right| \quad M_{2}(x)=\left|\begin{array}{cc}
\left(x^{2}-r^{2}\right) / r & -x / r \\
x / r & -1 / r
\end{array}\right| .
$$

Then, the energy $x$ is in the spectrum of $R_{l}$, if $\left|x_{l}(x)\right| \leqslant 1$ where

$$
x_{-1}=\frac{1}{2} \operatorname{Tr}\left(M_{1}\right), \quad x_{0}=\frac{1}{2} \operatorname{Tr}\left(M_{1} M_{2}\right), \quad x_{1}=\frac{1}{2} \operatorname{Tr}\left(M_{1} M_{2} M_{1} M_{2} M_{1}\right)
$$
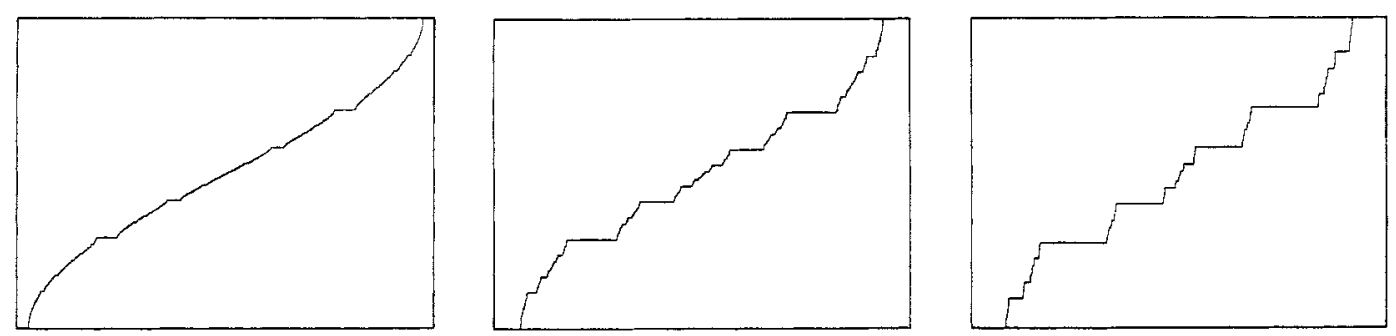

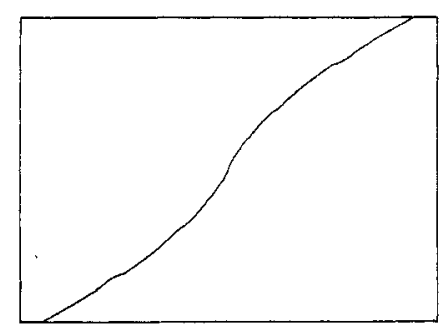

a)

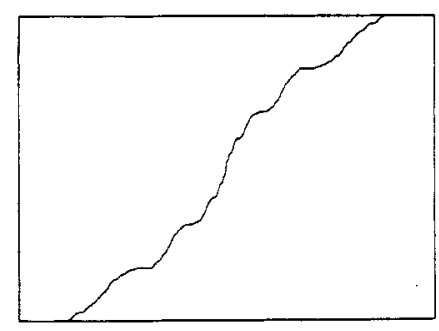

b)

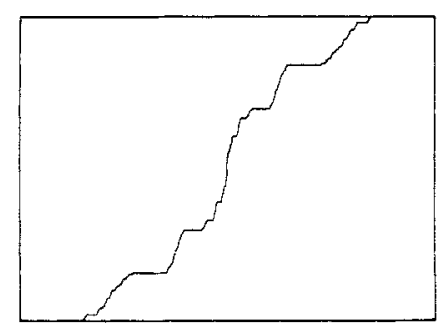

c)

Fig. 3. - We show, respectively, the IDOS of the Octonacci chain (up) and the IDOS of the labyrinth, for a) $r=0.8$ (no gap, finite measure), b) $r=0.6$ (some gaps and finite measure) and $c$ ) $r=0.3$ (infinity of gaps and zero measure). The energy varies between -2 and 2 , since $r<1$. 
and $x_{1}$ obeys the following recursion formula:

$$
x_{l+3}=4\left(x_{l+2}\right)^{2} x_{l+1}-\left(x_{l}+x_{l+2}\right) x_{l+2} / x_{l+1}-x_{l+1} .
$$

Taking into account this last result, and the derivation of sect. 3 , we are now able to calculate the DOS (density of state) for the labyrinth with hopping parameters $1, r, r^{2}$, for the three kinds of neighbouring sites. In fig. 3, we show the integrated DOS for the Octonacci chain and the labyrinth, for three typical values of $r$. Note that for $r$ close to 1 , the IDOS of the labyrinth resembles the IDOS of a square lattice, without any visible gap, whereas for small values of $r$, large gaps are appearing and the IDOS is a devil staircase. One can be interested in the measure of the spectrum. More precisely, for the Fibonacci chain, Kohmoto has found that when the size of the elementary cell increases, the measure of the spectrum vanishes like $F_{l}^{-\delta}$, where $F_{l}$ is the number of atoms in $R_{l}$. For the Octonacci chain, we obtain a similar result with

$$
F_{-1}=1, \quad F_{0}=1, \quad F_{l+2}=2 F_{l+1}+F_{l} .
$$

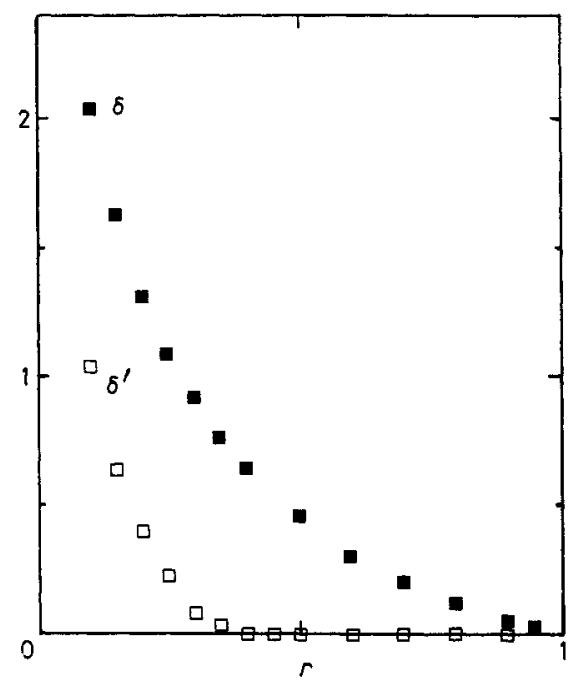

Fig. 4. $-\delta$ and $\delta "$ as a function of $r(0<r<1)$ for the "labyrinth».

For the labyrinth, we expect the measure of the spectrum to behave like $F_{l}^{-\delta^{\prime}}$. In fig. 4 , the dependence of $\delta$ and $\delta^{\prime \prime}$ with $r$ is shown for $0 \leqslant r \leqslant 1$. The first result is that although the DOS of the labyrinth is always singular, the measure of the spectrum is nonzero for $r>r_{0}$ with $r_{0} \approx 0.4$, zero otherwise. Moreover, there is an asymptotic relation between $\delta$ and $\delta^{\prime \prime}$. Indeed; for $r$ close to 0 , so that $\delta$ is large, each band of $R_{l}$ is very thin. Therefore, the "product» of the $F_{l}$ bands gives almost $F_{l}^{2}$ bands in the spectrum, so that the total bands width is about $F_{l}^{1-\delta}$. Hence, $\delta^{\prime \prime} \approx \delta-1$. Near $r_{0}$, a lot of bands overlap and this estimation is not correct.

One can illustrate the behaviour of the spectral measure, with a very simple example. Consider a real $k>3$, and the sets $C_{n}$ and $C$ defined by

$$
\left\{\begin{array}{l}
C_{n}=\left\{x /\left|y=\sum_{i=0}^{n-1} a_{i} / k^{i} \quad a_{i} \in\{-1,0,1\}\right|, \quad x \in\left[y-1 / k^{n}, y+1 / k^{n}\right]\right\} \\
C=\bigcap_{n=1}^{\infty} C_{n},
\end{array}\right.
$$


$C_{n}$ is a ramified set, and $C$ is a Cantor set. The measure of $C_{n}$ is $2 \times 3^{n} / k^{n}$. It goes to zero when $n$ goes to infinity like $3^{-n \delta}$, with $\delta=\ln (k) / \ln (3)-1$. Thus, the measure of $C$ is zero. Moreover, one can cover $C_{n}$ by means of $3^{n}$ segments of length $2 / k^{n}$. Thus, the Hausdorff dimension of $C$ is found to be $\lim _{n \rightarrow+\infty}\left[-\ln \left(3^{n}\right) / \ln \left(2 / k^{n}\right)\right]=\ln (3) / \ln (k)$. In general, the $p$ branches Cantor set has Hausdorff dimension $\ln (p) / \ln (k)$, and the corresponding $\delta$ is $\ln (k) / \ln (p)-1$. $C$ can modelize very grossly the spectrum of a 1D quasi-crystal (eventually with a different number of branches for an irrational number different from $\sqrt{2}-1$ or $(\sqrt{5}+1) / 2)$. In fact, it is known that instead of only one scaling factor $k$, there are an infinity of such ones [8] in the spectrum. Now, starting from $C$, we can build the two sets $C_{+}$and $C_{x}$ defined by $C_{+}=\{x+y \mid x, y \in C\}$ and $C_{x}=\{x y \mid x, y \in C\}$. Since $C_{+}$is a ramified Cantor set with 5 branches, the associated $\delta^{\prime \prime}$ is $\delta^{\prime \prime}=\ln (k) / \ln (5)-1$, if $k>5$, zero otherwise, while the Hausdorff dimension is $\ln 5 / \ln k$. Thus, for such a set, even if the IDOS can be shown to be singular, the measure can be finite, or zero, if the scaling factor $k$ becomes larger than 5 . The Result for $C_{x}$ is exactly the same. Thus, we find that the measure of $C_{+}$and $C_{x}$ becomes zero when $\delta$, the exponent related to $C$, is $\delta_{c}=\ln (5) / \ln (3)-1$. For a more general threebranches Cantor set, $C_{+}$and $C_{x}$ are expected to be two six-branches Cantor sets and $\delta_{c}=\ln (6) / \ln (3)-1 \approx 0.63093$. Even if this crude treatment cannot describe rigorously the behaviour of the spectrum of Fibonacci or «Octonacci» chains, it gives a reasonable value for $\delta_{c}$, and explains why the measure can be zero or not.

\section{Conclusion.}

In this letter, the excitation spectrum of a $2 \mathrm{D}$ quasi-periodic tiling very closely related to the standard octagonal tiling is calculated. The density of states is singular. No gap can be seen when the hopping parameters are too close to 1 . Moreover, the spectrum measure is finite for $r$ sufficiently close to 1 , and zero when $r$ is smaller than a critical value. Such a phenomenon can be explained by a very simple model describing the spectrum as a ramified Cantor set.

$$
* * *
$$

I am very indebted to R. Mosseri, J. Bellissard and M. Duneau for stimulating conversations.

\section{REFERENCES}

[1] Shechtman D., Blech I., Gratias D. and Cahn J. W., Phys. Rev. Lett., 53 (1984) 1951.

[2] Choy T. C., Phys. Rev. Lett., 55 (1985) 2915; Tsunetsugu H., Fujiwara T., Ueda K. and Tokihiro T., J. Phys. Soc. Jpn., 55 (1986) 1420.

[3] AUbry S. and ANDré G., Ann. Israel Phys. Soc., 3 (1980) 133.

[4] Kohmoto M., Kadanoff L. P. and Tang C., Phys. Rev. Lett., 50 (1983) 1870; Ostlund S., PanditT R., Rand D., Schellnhuber H. J. and Siggia E. D., Phys. Rev. Lett., 50 (1983) 1873.

[5] Kalugin P. A., Kitaev A. Yu. and Levitov L. S., Ž. Ėksp. Teor. Fiz., 91 (1986) 692.

[6] Ueda K. and Tsunetsugu H., Phys. Rev. Lett., 58 (1987) 1272.

[7] Sire C., Mosseri R. and Sadoc J. F., to be published in J. Phys. (Paris).

[8] LUCK J. M. and Niewenhuizen T. M., Europhys. Lett., 2 (1986) 257. 